"Mircea cel Batran" Naval Academy Scientific Bulletin, Volume XIX - 2016 - Issue 2

The journal is indexed in: PROQUEST / DOAJ / Crossref / EBSCOhost / INDEX COPERNICUS / DRJI / OAJI I JOURNAL INDEX I I2OR / SCIENCE LIBRARY INDEX / Google Scholar / Academic Keys/ ROAD Open Access I Academic Resources / Scientific Indexing Services / SCIPIO / JIFACTOR

\title{
CONSIDERATIONS ON THE CALCULATION OF THE MAIN CHARACTERISTICS OF THE SHIP SEAKEEPING
}

\author{
Valentin ONCICA ${ }^{1}$ \\ Alecu TOMA ${ }^{2}$ \\ Ionut-Cristian SCURTU ${ }^{3}$
}

${ }^{1}$ Lecturer, Eng., PhD, „Mircea cel Batran” Naval Academy, Constanta, Romania, valentin.oncica@anmb.ro

${ }^{2}$ Lecturer, Eng., PhD, „Mircea cel Batran” Naval Academy, Constanta, Romania, alecu.toma@anmb.ro

${ }^{3}$ Instr., Eng., PhD, „Mircea cel Batran” Naval Academy, Constanta, Romania, ionut.scurtu@anmb.ro

\begin{abstract}
The motion of a ship or floating structure is important for determining of the dynamic load on the crew/ passengers, structural materials and equipment, and cargo. In this paper we propose to determine the parameters that influence heaving and pitching amplitude relevant to study the 97,000 dwt bulk carrier. With equations based on regression analysis can be estimated amplitude values for various degrees of sea agitation and for various loading situations of the ship. The results are useful for determining additional loads induced both structural elements and the components of equipment and installations on board ships.
\end{abstract}

Keywords: heaving, pitching, regression analysis

\section{INTRODUCTION}

Presented model in fig.1. will be used in Autoship parameter calculus.

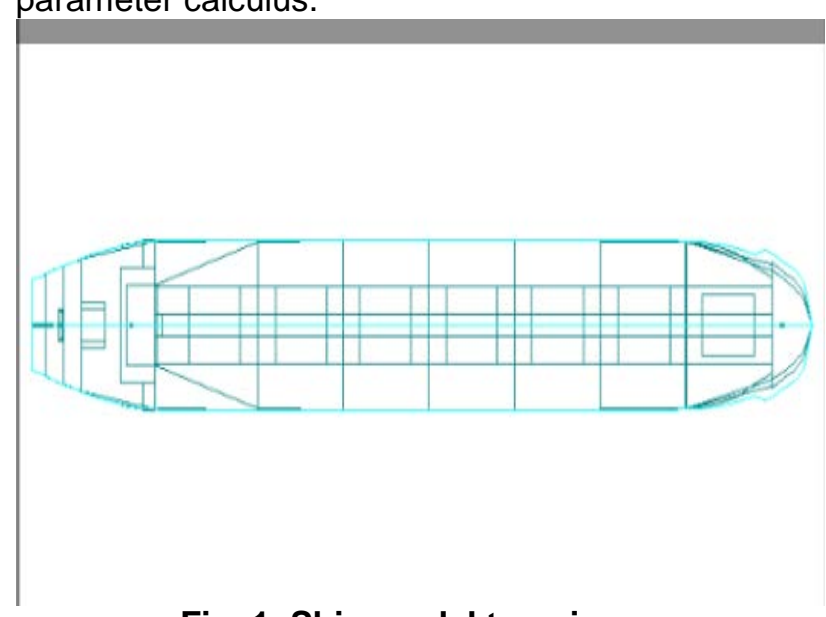

Fig. 1. Ship model top view

As a rigid body, a ship has six degrees of freedom: three translations (heave, surge and sway) and three rotations (roll, pitching and yaw). In this paper we deal with heaving and pitching motions, becouse they are very important for discomfort of passengers and crew, and for operation of machines and installations.

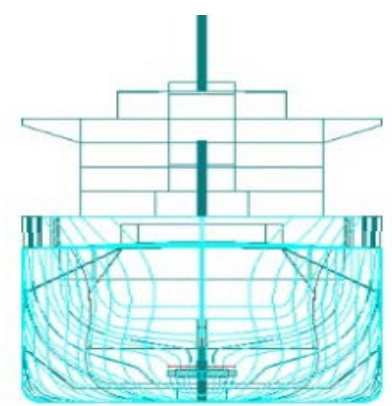

Fig. 2 Ship model bow view

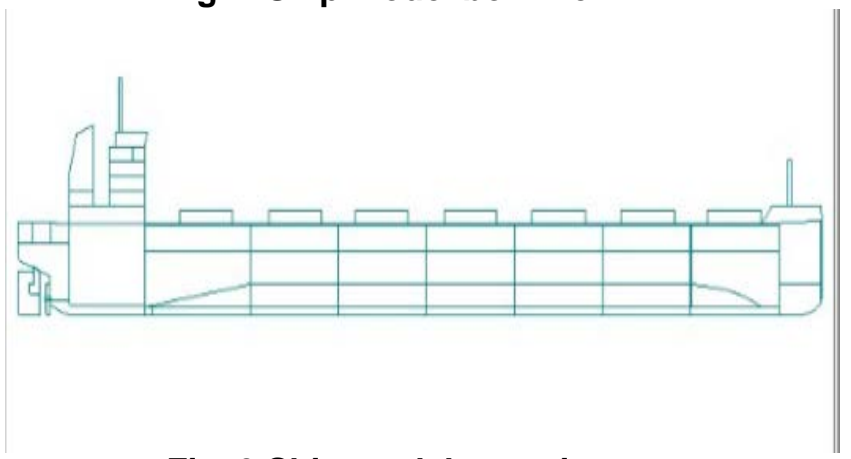

Fig. 3 Ship model port view

With the data obtained from various seakeeping tests, were made statistical analyses as function of different parametric values. One of these methods is multiple regression analyses [1] by which we can estimate heaving and pitching amplitude in regular seas as function of ship dimensions $(L, B, d)$, waterplane area coefficient $\left(C_{W}\right)$, block coefficient $\left(C_{B}\right)$, buoyancy center $\left(x_{B}\right)$, inertial characteristics of the ship $\left(k_{y y}\right)$, ship speed $(v)$ and sea state (Beaufort Number). 
"Mircea cel Batran" Naval Academy Scientific Bulletin, Volume XIX - 2016 - Issue 2 The journal is indexed in: PROQUEST / DOAJ / Crossref / EBSCOhost / INDEX COPERNICUS / DRJI / OAJI I JOURNAL INDEX I I2OR / SCIENCE LIBRARY INDEX / Google Scholar / Academic Keys/ ROAD Open Access I Academic Resources / Scientific Indexing Services / SCIPIO I JIFACTOR

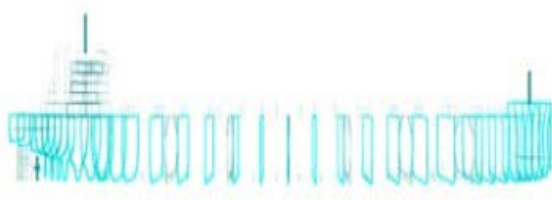

Fig. 4. Ship model frames view

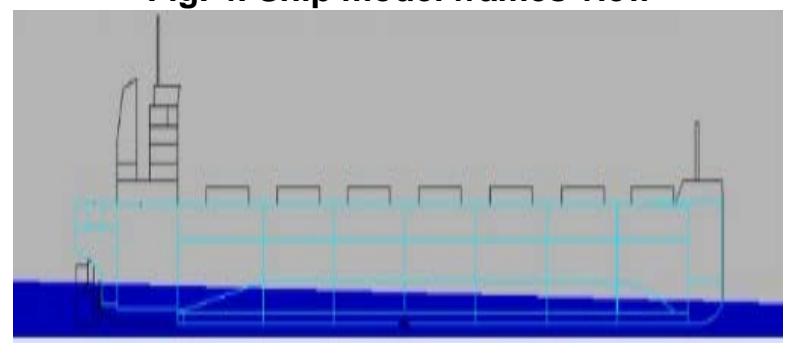

Fig. 5. Ship model floating in Autohydro-1

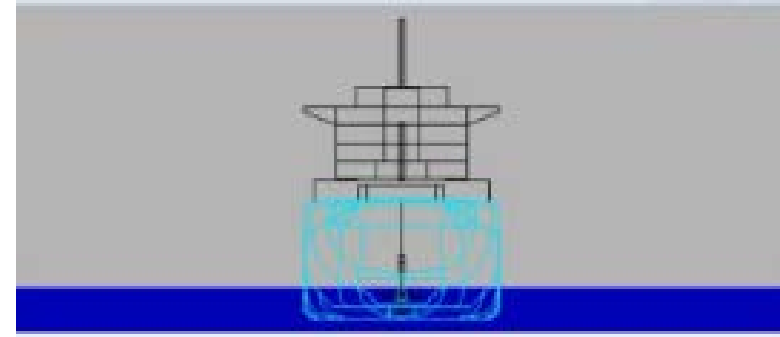

Fig. 6. Ship model floating in Autohydro-2

Equations for the estimation of heaving and pitching amplitude in regular seas, with multiple regression analyses are [1] below (1) and (2) :

$z_{a}=A_{0}+A_{1} \times C_{W}+A_{2} \times C_{B}+A_{3} \times \frac{L}{B}+A_{4}$.

$\left(\frac{L}{d}\right)^{-1}+A_{5} \times 100 \times \frac{x_{B}}{L}+A_{6} \times \frac{k_{y y}}{L}+A_{7} \times \frac{v}{\sqrt{L}}$

$\theta_{a}=B_{0}+B_{1} \times C_{W}+B_{2} \times C_{B}+B_{3} \times \frac{L}{B}+B_{4} \times$

$\frac{L}{d}+B_{5} \times 100 \times \frac{x_{B}}{L}+B_{6} \times \frac{k_{y y}}{L}+B_{7} \times \frac{v}{\sqrt{L}}+B_{8}$.

$\left(\frac{v}{\sqrt{L}}\right)^{2}$

where the coefficients $A_{0} \div A_{7}$ and $B_{0} \div B_{8}$ are given in Table $1 \div 6$ as functions of length of the ship and Beaufort Number; $C_{W}=$ waterplane area coefficient; $C_{B}=$ block coefficient; $L[\mathrm{~m}]=$ length of the ship; $B[\mathrm{~m}]=$ beam of the ship; $d[m]=$ draft of the ship; $x_{B}[m]=$ abscissa of

buoyancy center; $k_{y y}[\mathrm{~m}]=$ radius of gyration about $\mathrm{y}$-axis; $v$ [knots] $=$ ship speed.

$$
\begin{aligned}
& a=A_{0}+A_{1} \times C_{W}+A_{2} \times C_{B}+A_{3} \times \frac{L}{B}+A_{4} \times \\
& \left(\frac{L}{d}\right)^{-1}+A_{5} \times 100 \times \frac{x_{B}}{L}+A_{6} \times \frac{k_{y y}}{L} \\
& b=B_{0}+B_{1} \times C_{W}+B_{2} \times C_{B}+B_{3} \times \frac{L}{B}+B_{4} \times \\
& \frac{L}{d}+B_{5} \times 100 \times \frac{x_{B}}{L}+B_{6} \times \frac{k_{y y}}{L}
\end{aligned}
$$

relationships 1 and 2 are as follows:

$$
\begin{aligned}
& z_{a}=a+A_{7} \times \frac{v}{\sqrt{L}} \\
& \theta_{\alpha}=b+B_{7} \times \frac{v}{\sqrt{L}}+B_{8} \times\left(\frac{v}{\sqrt{L}}\right)^{2}
\end{aligned}
$$

Relation (5) shows that the heaving amplitude is a $1^{\text {st }}$ grade function in $\frac{v}{\sqrt{L}}$, while relation (6) shows that the pitching amplitude is a $2^{\text {nd }}$ grade function

\begin{tabular}{|c|c|c|c|c|c|c|c|c|c|}
\hline $\begin{array}{c}\text { Beau } \\
\text { fort } \\
\text { Num } \\
\text { ber }\end{array}$ & $B_{0}$ & $B_{1}$ & $B_{2}$ & $B_{3}$ & $B_{4}$ & $B_{5}$ & $B_{6}$ & $B_{7}$ & $B_{8}$ \\
\hline 5 & $\begin{array}{l}2 . \\
21\end{array}$ & $\begin{array}{c}- \\
4.2 \\
1 \\
\end{array}$ & $\begin{array}{c}2.2 \\
1\end{array}$ & $\begin{array}{c}0.0 \\
9\end{array}$ & $\begin{array}{c}- \\
0.00 \\
55\end{array}$ & $\begin{array}{c}- \\
0.0 \\
35 \\
\end{array}$ & $\begin{array}{l}- \\
1 . \\
4\end{array}$ & $\begin{array}{c}0 . \\
95\end{array}$ & $\begin{array}{l}- \\
0 . \\
46\end{array}$ \\
\hline 6 & $\begin{array}{c}3 . \\
86\end{array}$ & $\begin{array}{c}- \\
8.8 \\
8 \\
\end{array}$ & $\begin{array}{c}4.7 \\
5\end{array}$ & $\begin{array}{l}0.1 \\
72\end{array}$ & $\begin{array}{c}-\overline{0} \\
0.01 \\
75\end{array}$ & $\begin{array}{c}- \\
0.0 \\
62 \\
\end{array}$ & $\begin{array}{c}1 . \\
6\end{array}$ & $\begin{array}{l}2 . \\
13\end{array}$ & $\begin{array}{l}- \\
0 . \\
94\end{array}$ \\
\hline 7 & $\begin{array}{c}5 . \\
01\end{array}$ & $\begin{array}{c}- \\
14 . \\
38 \\
\end{array}$ & $\begin{array}{c}7.9 \\
1\end{array}$ & $\begin{array}{l}0.2 \\
52\end{array}$ & $\begin{array}{c}- \\
0.03 \\
56\end{array}$ & $\begin{array}{c}- \\
0.0 \\
85 \\
\end{array}$ & $\begin{array}{c}4 . \\
9\end{array}$ & $\begin{array}{l}4 . \\
13\end{array}$ & $\begin{array}{l}- \\
1 . \\
51\end{array}$ \\
\hline 8 & $\begin{array}{l}5 . \\
98\end{array}$ & $\begin{array}{c}- \\
20 . \\
52\end{array}$ & $\begin{array}{l}11 . \\
57\end{array}$ & $\begin{array}{c}0.3 \\
05\end{array}$ & $\begin{array}{c}- \\
0.05 \\
92\end{array}$ & $\begin{array}{c}- \\
0.1 \\
05\end{array}$ & $\begin{array}{c}12 \\
.9\end{array}$ & $\begin{array}{l}5 . \\
14\end{array}$ & $\begin{array}{l}- \\
2 . \\
01\end{array}$ \\
\hline
\end{tabular}
in $\frac{v}{\sqrt{L}}$, where $a$ and $b$ can be found in Table 3 , for each of the three load cases considered.

Table 1

\begin{tabular}{|c|c|c|c|c|c|c|c|c|}
\hline $\begin{array}{c}\text { Beauf } \\
\text { ort } \\
\begin{array}{c}\text { Numb } \\
\text { er }\end{array}\end{array}$ & $A_{0}$ & $A_{1}$ & $A_{2}$ & $A_{3}$ & $A_{4}$ & $A_{5}$ & $A_{6}$ & $A_{7}$ \\
\hline 5 & 2.3 & $\begin{array}{c}5.8 \\
4\end{array}$ & $\begin{array}{c}0.8 \\
2\end{array}$ & $\begin{array}{c}0.1 \\
52\end{array}$ & $\begin{array}{c}17 . \\
4\end{array}$ & $\begin{array}{c}0.0 \\
94\end{array}$ & 7.5 & $\begin{array}{c}0.3 \\
9\end{array}$ \\
\hline 6 & 2.9 & $\begin{array}{c}13 . \\
7\end{array}$ & 2.8 & $\begin{array}{c}0.2 \\
43\end{array}$ & $\begin{array}{c}55 . \\
39\end{array}$ & $\begin{array}{c}0.2 \\
14\end{array}$ & $\begin{array}{c}19 . \\
27\end{array}$ & $\begin{array}{c}0.8 \\
9\end{array}$ \\
\hline 7 & 3.0 & $\begin{array}{c}21 . \\
6\end{array}$ & 5.5 & 0.3 & 107 & 0.3 & 32. & 2.2 \\
29 & 6 & 29 & 57 & 73 & 4 \\
\hline 8 & 3.0 & $\begin{array}{c}31 . \\
5\end{array}$ & 9.6 & $\begin{array}{c}0.3 \\
27\end{array}$ & $\begin{array}{c}171 \\
.6\end{array}$ & $\begin{array}{c}0.5 \\
07\end{array}$ & $\begin{array}{c}49 . \\
3\end{array}$ & $\begin{array}{c}4.1 \\
8\end{array}$ \\
\hline
\end{tabular}

Table 2 
"Mircea cel Batran" Naval Academy Scientific Bulletin, Volume XIX - 2016 - Issue 2 The journal is indexed in: PROQUEST / DOAJ / Crossref / EBSCOhost / INDEX COPERNICUS / DRJI / OAJI I JOURNAL INDEX I I2OR / SCIENCE LIBRARY INDEX / Google Scholar / Academic Keys/ ROAD Open Access I Academic Resources / Scientific Indexing Services / SCIPIO / JIFACTOR

Table 3

\begin{tabular}{|c|c|c|c|c|c|c|}
\hline \multirow{2}{*}{$\begin{array}{l}\text { Beaufort } \\
\text { Number }\end{array}$} & \multicolumn{2}{|c|}{ case 1} & \multicolumn{2}{|c|}{ case 2} & \multicolumn{2}{|c|}{ case 3} \\
\hline & $a$ & $b$ & $a$ & $b$ & $a$ & $b$ \\
\hline 5 & 1.649079 & 0.250105 & 1.187617 & 0.024243 & 0.72172 & -0.16262 \\
\hline 6 & 3.181047 & 0.748633 & 1.744736 & 0.206545 & 0.290972 & -0.30823 \\
\hline 7 & 5.08345 & 0.470576 & 2.321076 & -0.50335 & -0.48292 & -1.50501 \\
\hline 8 & 7.698549 & 0.761416 & 3.238125 & -0.74133 & -1.29136 & -2.36567 \\
\hline
\end{tabular}

Ship data:

Caz 1: $C_{W}=0.921 ; C_{B}=0.839 ; \frac{L}{B}=6.284$;

$\frac{L}{d}=16.67 ; \frac{x_{B}}{L}=1.73 ; \frac{k_{y y}}{L}=0.25$

Caz 2: $C_{W}=0.91 ; C_{B}=0.71 ; \frac{L}{B}=6.284$;

$\frac{L}{d}=23.103 ; \frac{x_{B}}{L}=0.35 ; \frac{k_{y y}}{L}=0.25$

Caz 3: $C_{W}=0.878 ; C_{B}=0.552 ; \frac{L}{B}=6.284$;

$\frac{L}{d}=35.649 ; \frac{x_{B}}{L}=-2.41 ; \frac{k_{y y}}{L}=0.25$

Graphics: $z_{a}=f\left(\frac{v}{\sqrt{L}}\right)$ si $\theta_{a}=f\left(\frac{v}{\sqrt{L}}\right)$, forcele 4

Beaufort scale and tree load cases.

\section{Hull Data (with appendages)}

Baseline Draft: 14.097 at Origin

Trim: $\quad 0.01 \mathrm{deg}$.

Heel: zero

\section{DIMENSIONS}

Length Overall: $238.800 \mathrm{~m} \quad$ LWL: $238.800 \mathrm{~m}$ Beam: $38.000 \mathrm{~m}$ BWL: $38.000 \mathrm{~m}$

Volume: $107512.200 \mathrm{~m}^{3}$ Displacement:

110200.600 MT

\section{COEFFICIENTS}

Prismatic: 0.843 Block: 0.839 Midship:

0.995 Waterplane: 0.921

\section{RATIOS}

Length/Beam: 6.284 Displacement/length:

225.528 Beam/Depth: 2.691

MT/ cm Immersion: 85.651

\section{AREAS}

Waterplane: $8356.173 \mathrm{~m}^{2}$ Wetted Surface:

$13756.710 \mathrm{~m}^{2}$

Under Water Lateral Plane: $3348.244 \mathrm{~m}^{2}$

Above Water Lateral Plane: $2287.907 \mathrm{~m}^{2}$

CENTROIDS (Meters)

Buoyancy: $\mathrm{LCB}=4.071$ fwd $\quad$ TCB $=0.000$

port $\quad \mathrm{VCB}=7.347$

Flotation: $\mathrm{LCF}=3.770 \mathrm{aft}$
Under Water LP: 1.928 aft of Origin, 7.013 below waterline.

Above Water LP: 11.979 aft of Origin, 6.278 above waterline.

Note: Coefficients calculated based on waterline length at given draft

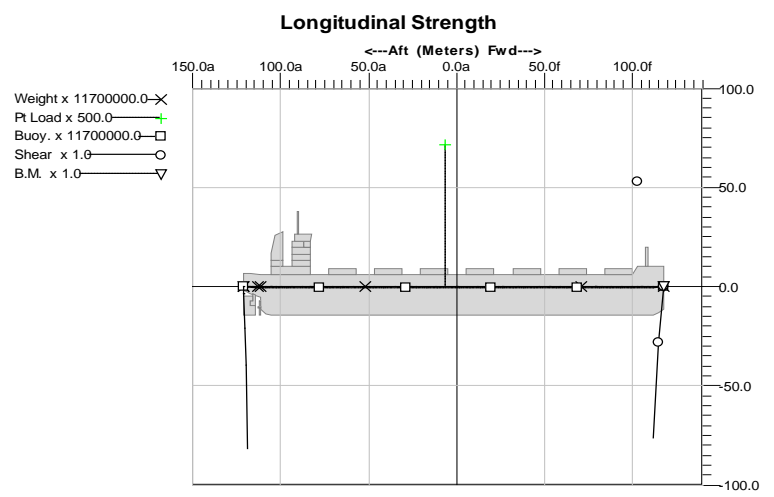

Fig. 7. Ship model results in Autohydro

* Point weight in Metric Tons

\begin{tabular}{|l|r|}
\hline Max. Shear & 18161.48 \\
& MT \\
\hline Max. Bending Moment & -1033757 \\
& MT-m \\
\hline
\end{tabular}

Righting Arms vs. Heel

Righting Arms vs Heel Angle

\begin{tabular}{|r|r|}
\hline $\begin{array}{c}\text { Heel Angle } \\
\text { (deg) }\end{array}$ & $\begin{array}{c}\text { Righting Arm } \\
(\mathrm{m})\end{array}$ \\
\hline 0.00 & 0.000 \\
\hline $5.00 \mathrm{~s}$ & 0.643 \\
\hline $10.00 \mathrm{~s}$ & 1.298 \\
\hline $15.00 \mathrm{~s}$ & 1.977 \\
\hline $20.00 \mathrm{~s}$ & 2.657 \\
\hline $25.00 \mathrm{~s}$ & 3.173 \\
\hline $30.00 \mathrm{~s}$ & 3.549 \\
\hline $35.00 \mathrm{~s}$ & 3.833 \\
\hline $40.00 \mathrm{~s}$ & 4.032 \\
\hline $45.00 \mathrm{~s}$ & 4.132 \\
\hline $47.47 \mathrm{~s}$ & 4.144 \\
\hline $50.00 \mathrm{~s}$ & 4.132 \\
\hline $55.00 \mathrm{~s}$ & 4.046 \\
\hline $60.00 \mathrm{~s}$ & 3.892 \\
\hline &
\end{tabular}


"Mircea cel Batran" Naval Academy Scientific Bulletin, Volume XIX - 2016 - Issue 2 The journal is indexed in: PROQUEST / DOAJ / Crossref / EBSCOhost / INDEX COPERNICUS / DRJI / OAJI I JOURNAL INDEX I I2OR / SCIENCE LIBRARY INDEX / Google Scholar / Academic Keys/ ROAD Open Access I Academic Resources / Scientific Indexing Services / SCIPIO / JIFACTOR

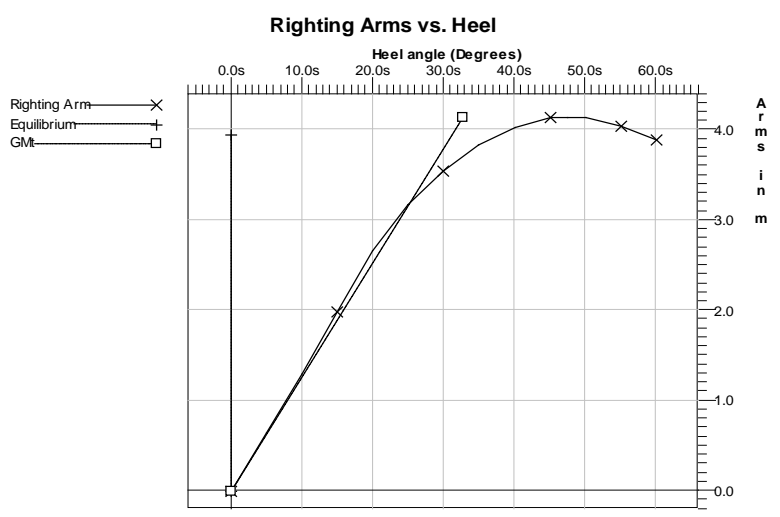

Fig. 8 Ship model righting arm results in Autohydro

\section{Hull Data (with appendages)}

Baseline Draft: 10.172 at Origin

Trim: aft $0.82 \mathrm{deg}$.

Heel: zero

\section{DIMENSIONS}

Length Overall: $238.800 \mathrm{~m} \quad$ LWL: $238.700 \mathrm{~m}$

Beam: $38.000 \mathrm{~m} \quad$ BWL: $38.000 \mathrm{~m}$

Volume: $75257.480 \mathrm{~m} 3$ Displacement:

$77139.330 \mathrm{MT}$

\section{COEFFICIENTS}

Prismatic: 0.783

0.907 Waterplane: 0.910

Block: $0.710 \quad$ Midship:

\section{RATIOS}

Length/Beam: 6.284 Displacement/length:

158.066 Beam/Depth: 3.250

MT/ cm Immersion: 84.628

\section{AREAS}

Waterplane: $8256.372 \mathrm{~m} 2$ Wetted Surface:

$11778.250 \mathrm{~m} 2$

Under Water Lateral Plane: $2418.640 \mathrm{~m} 2$

Above Water Lateral Plane: $3217.510 \mathrm{~m} 2$

\section{CENTROIDS (Meters)}

Buoyancy: $\mathrm{LCB}=0.831$ fwd $\quad$ TCB $=0.000$

port $\quad \mathrm{VCB}=5.330$

Flotation: $L C F=2.259$ aft

Under Water LP: 8.684 aft of Origin, 5.111 below waterline.

Above Water LP: 4.304 aft of Origin, 7.732

above waterline.

Note: Coefficients calculated based on waterline length at given draft

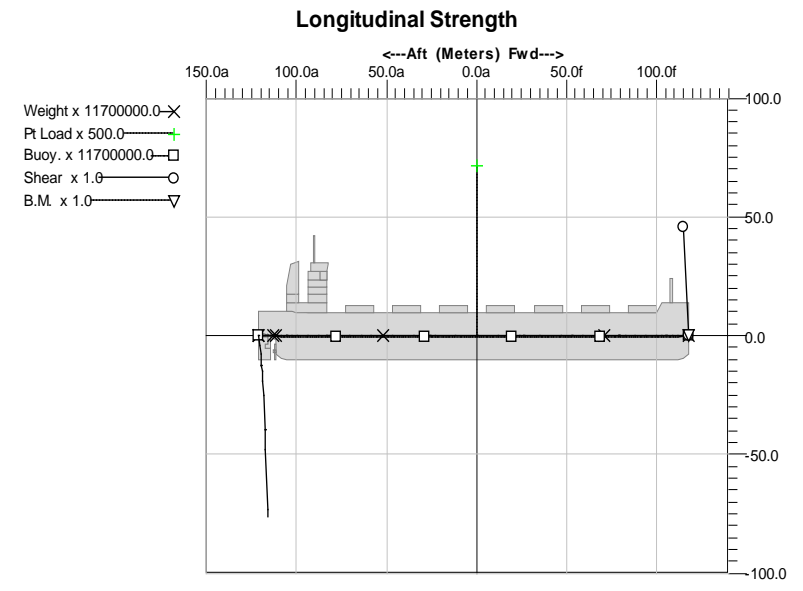

* Point weight in Metric Tons

Fig. 9. Ship model righting arm in Autohydro

Max. Shear $\quad-18517.47 \mathrm{MT}$ at 0.000

Max. Bending Moment -946189 MT- at 0.000 $\mathrm{m}$

Righting Arms vs Heel Angle

\begin{tabular}{|l|l|}
\hline $\begin{array}{l}\text { Heel Angle } \\
(\text { deg})\end{array}$ & $\begin{array}{l}\text { Righting } \\
\text { Arm } \\
(\mathrm{m})\end{array}$ \\
\hline 0.00 & 0.000 \\
\hline $5.00 \mathrm{~s}$ & 0.452 \\
\hline $10.00 \mathrm{~s}$ & 0.908 \\
\hline $15.00 \mathrm{~s}$ & 1.425 \\
\hline $20.00 \mathrm{~s}$ & 2.081 \\
\hline $25.00 \mathrm{~s}$ & 2.879 \\
\hline $30.00 \mathrm{~s}$ & 3.720 \\
\hline $35.00 \mathrm{~s}$ & 4.300 \\
\hline $40.00 \mathrm{~s}$ & 4.624 \\
\hline $45.00 \mathrm{~s}$ & 4.786 \\
\hline $50.00 \mathrm{~s}$ & 4.835 \\
\hline $50.19 \mathrm{~s}$ & 4.835 \\
\hline $55.00 \mathrm{~s}$ & 4.801 \\
\hline $60.00 \mathrm{~s}$ & 4.698 \\
\hline
\end{tabular}

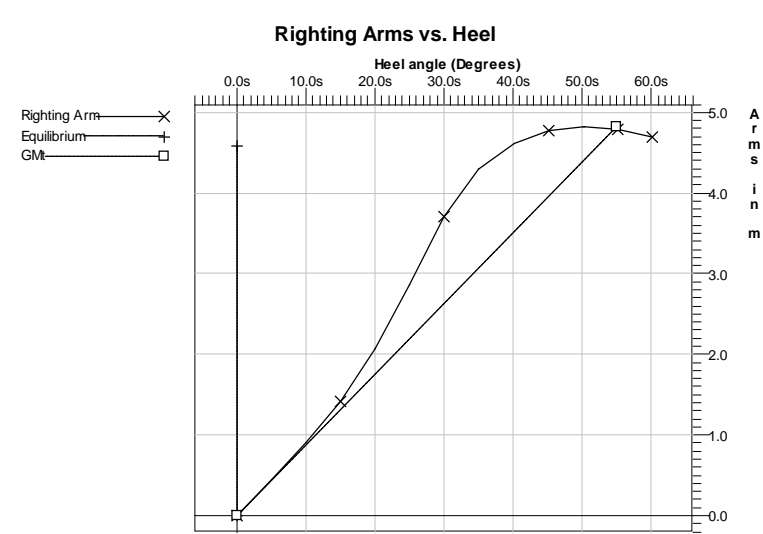

Fig. 10. Ship model righting arm results graph 
"Mircea cel Batran" Naval Academy Scientific Bulletin, Volume XIX - 2016 - Issue 2 The journal is indexed in: PROQUEST / DOAJ / Crossref / EBSCOhost / INDEX COPERNICUS / DRJI / OAJI I JOURNAL INDEX I I2OR / SCIENCE LIBRARY INDEX / Google Scholar / Academic Keys/ ROAD Open Access I Academic Resources / Scientific Indexing Services / SCIPIO / JIFACTOR

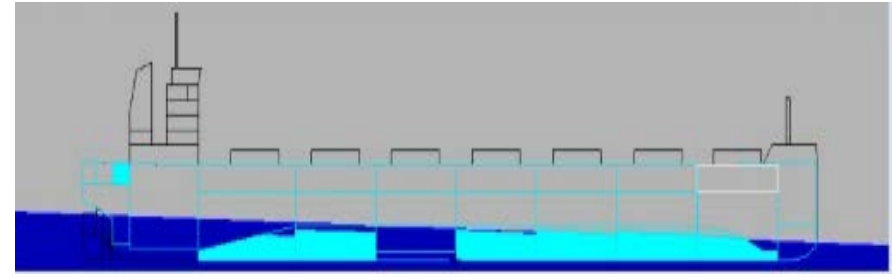

Fig. 11. Ship model floating case in Autohydro

\section{Hull Data (with appendages)}

Baseline Draft: 6.592 at Origin

Trim: aft 1.43 deg.

Heel: zero

\section{DIMENSIONS}

Length Overall: $238.800 \mathrm{~m} \quad$ LWL: $238.643 \mathrm{~m}$ Beam: $38.000 \mathrm{~m} \quad$ BWL: $38.000 \mathrm{~m}$

Volume: $46234.510 \mathrm{~m}^{3}$ Displacement:

\subsection{MT}

\section{COEFFICIENTS}

Prismatic: $0.678 \quad$ Block: 0.552 Midship:

0.814 Waterplane: 0.878

\section{RATIOS}

Length/Beam: 6.284 Displacement/length: 97.178 Beam/Depth: 4.116

MT/ cm Immersion: 81.651

\section{AREAS}

Waterplane: $7965.938 \mathrm{~m}^{2}$ Wetted Surface: $9975.805 \mathrm{~m}^{2}$

Under Water Lateral Plane: $1576.405 \mathrm{~m}^{2}$

Above Water Lateral Plane: $4059.747 \mathrm{~m}^{2}$

\section{CENTROIDS (Meters)}

Buoyancy: $\mathrm{LCB}=5.674$ aft $\quad$ TCB $=0.000$

stbd $\quad \mathrm{VCB}=3.545$

Flotation: $L C F=1.042 \mathrm{fwd}$

Under Water LP: 20.500 aft of Origin, 3.513

below waterline.

Above Water LP: 0.806 aft of Origin, 9.317

above waterline.

Note: Coefficients calculated based on waterline length at given draft

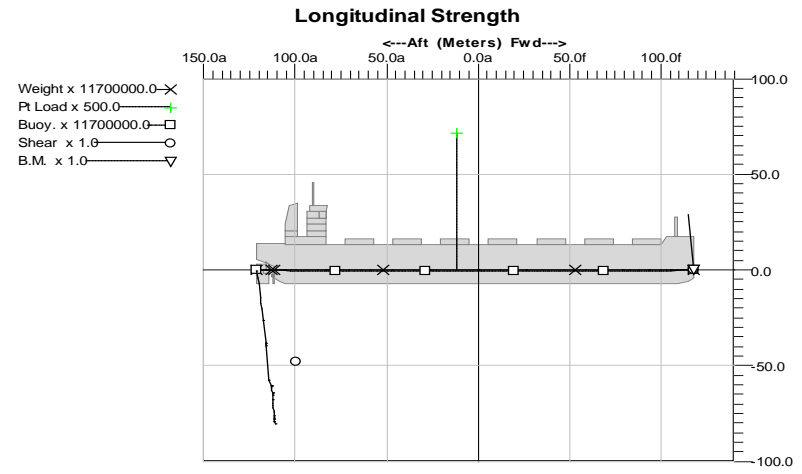

Fig. 12. Ship model BM results in Autohydro

\begin{tabular}{|c|c|c|}
\hline $\begin{array}{c}\text { Location } \\
(\mathrm{m})\end{array}$ & $\begin{array}{l}\text { Shear } \\
\text { (MT) }\end{array}$ & $\begin{array}{c}\text { Bending } \\
(\mathrm{MT}-\mathrm{m})\end{array}$ \\
\hline $117.443 f$ & 0.00 & \\
\hline \multicolumn{3}{|l|}{$117.443 f$} \\
\hline $117.400 f$ & 0.00 & \\
\hline $114.563 f$ & 29.24 & -41 \\
\hline $111.625 f$ & 124.11 & -262 \\
\hline $108.687 f$ & 281.73 & -857 \\
\hline $105.750 f$ & 491.08 & -1993 \\
\hline $104.760 f$ & 571.73 & -2523 \\
\hline \multicolumn{3}{|l|}{ 104.760f } \\
\hline $102.812 f$ & 694.55 & -3764 \\
\hline $99.875 f$ & 896.95 & -6108 \\
\hline $94.000 f$ & 1352.03 & -12715 \\
\hline $92.845 f$ & 1446.89 & -14336 \\
\hline $88.125 f$ & 1844.10 & -22118 \\
\hline $82.250 f$ & 2361.11 & -34484 \\
\hline $78.600 f$ & 2696.19 & -43726 \\
\hline \multicolumn{3}{|l|}{$78.600 f$} \\
\hline $70.500 f$ & 3489.03 & -68750 \\
\hline $65.500 \mathrm{f}$ & 4015.04 & -87525 \\
\hline $58.750 f$ & 4751.52 & -117125 \\
\hline $52.440 f$ & 5465.88 & -149376 \\
\hline \multicolumn{3}{|l|}{$52.440 f$} \\
\hline $52.400 f$ & 5467.00 & -149595 \\
\hline \multicolumn{3}{|l|}{$52.400 f$} \\
\hline $47.000 f$ & 6109.55 & -180864 \\
\hline $35.250 f$ & 7598.10 & -261326 \\
\hline $26.280 f$ & 8818.83 & -334940 \\
\hline \multicolumn{3}{|l|}{$26.280 f$} \\
\hline $23.500 f$ & 9258.33 & -360078 \\
\hline $11.750 f$ & 11193.69 & -480162 \\
\hline $0.120 f$ & 13233.93 & -622139 \\
\hline \multicolumn{3}{|l|}{$0.120 f$} \\
\hline 0.000 & 13264.50 & -623730 \\
\hline $11.750 \mathrm{a}$ & 16325.03 & -797490 \\
\hline $12.000 \mathrm{a}$ & -19597.18 & -801580 \\
\hline $23.500 a$ & -16471.29 & -594117 \\
\hline $26.040 \mathrm{a}$ & -15764.40 & -553188 \\
\hline
\end{tabular}


"Mircea cel Batran" Naval Academy Scientific Bulletin, Volume XIX - 2016 - Issue 2 The journal is indexed in: PROQUEST / DOAJ / Crossref / EBSCOhost / INDEX COPERNICUS / DRJI / OAJI I JOURNAL INDEX I I2OR / SCIENCE LIBRARY INDEX / Google Scholar / Academic Keys/ ROAD Open Access I Academic Resources / Scientific Indexing Services / SCIPIO / JIFACTOR

\begin{tabular}{|l|r|r|}
\hline $26.040 a$ & & \\
\hline $35.250 a$ & -13804.85 & -416986 \\
\hline $47.000 a$ & -11182.81 & -270119 \\
\hline $52.200 a$ & -9993.52 & -215082 \\
\hline $52.200 a$ & & \\
\hline $58.750 a$ & -8445.65 & -154697 \\
\hline $63.891 a$ & -7216.79 & -114463 \\
\hline $70.500 a$ & -5642.77 & -71997 \\
\hline $76.375 a$ & -4271.14 & -42928 \\
\hline $82.250 a$ & -2977.10 & -21711 \\
\hline $83.590 a$ & -2704.82 & -17913 \\
\hline $83.590 a$ & & \\
\hline $88.125 a$ & -1796.53 & -7753 \\
\hline $94.000 a$ & -790.32 & -301 \\
\hline $99.875 a$ & -47.55 & 1997 \\
\hline $102.812 a$ & 214.37 & 1720 \\
\hline $105.750 a$ & 404.15 & 783 \\
\hline $106.000 a$ & 417.27 & 679 \\
\hline $106.000 a$ & & -250 \\
\hline $108.687 a$ & 194.86 & -166 \\
\hline $111.440 a$ & -80.02 & -347 \\
\hline $111.440 a$ & & -256 \\
\hline $111.600 a$ & -79.02 & -335 \\
\hline $111.625 a$ & -78.86 & -333 \\
\hline $111.844 a$ & -77.50 & -317 \\
\hline $111.854 a$ & -77.44 & -316 \\
\hline $111.879 a$ & -77.23 & -314 \\
\hline $111.978 a$ & -75.99 & -307 \\
\hline $111.982 a$ & -75.93 & -307 \\
\hline $112.109 a$ & -73.88 & -298 \\
\hline $112.126 a$ & -73.60 & -296 \\
\hline $112.236 a$ & -71.71 & -289 \\
\hline $112.300 a$ & -70.61 & -285 \\
\hline $112.364 a$ & -69.51 & -280 \\
\hline $112.474 a$ & -67.65 & -273 \\
\hline $112.491 a$ & -67.38 & -272 \\
\hline $112.618 a$ & -65.40 & -264 \\
\hline $112.622 a$ & -65.35 & -264 \\
\hline $112.721 a$ & -64.19 & -258 \\
\hline $112.746 a$ & -64.01 & -257 \\
\hline $112.756 a$ & -63.95 & -256 \\
\hline $113.000 a$ & -62.76 & -242 \\
\hline & & \\
\hline
\end{tabular}

\begin{tabular}{|l|l|l|}
\hline $113.317 a$ & -61.42 & -223 \\
\hline $113.476 a$ & -60.85 & -214 \\
\hline $113.555 a$ & -60.59 & -210 \\
\hline $113.595 a$ & -60.46 & -208 \\
\hline
\end{tabular}

* Point weight in Metric Tons

Righting Arms vs Heel Angle

\begin{tabular}{|r|r|}
\hline $\begin{array}{c}\text { Heel Angle } \\
\text { (deg) }\end{array}$ & $\begin{array}{c}\text { Rightin } \\
\text { g Arm } \\
(\mathrm{m})\end{array}$ \\
\hline 0.00 & 0.000 \\
\hline $5.00 \mathrm{~s}$ & 1.554 \\
\hline $10.00 \mathrm{~s}$ & 3.120 \\
\hline $15.00 \mathrm{~s}$ & 4.688 \\
\hline $20.00 \mathrm{~s}$ & 6.152 \\
\hline $25.00 \mathrm{~s}$ & 7.365 \\
\hline $30.00 \mathrm{~s}$ & 8.299 \\
\hline $35.00 \mathrm{~s}$ & 9.030 \\
\hline $40.00 \mathrm{~s}$ & 9.594 \\
\hline $45.00 \mathrm{~s}$ & 9.961 \\
\hline $50.00 \mathrm{~s}$ & 10.110 \\
\hline $51.24 \mathrm{~s}$ & 10.116 \\
\hline $55.00 \mathrm{~s}$ & 10.066 \\
\hline $60.00 \mathrm{~s}$ & 9.861 \\
\hline &
\end{tabular}

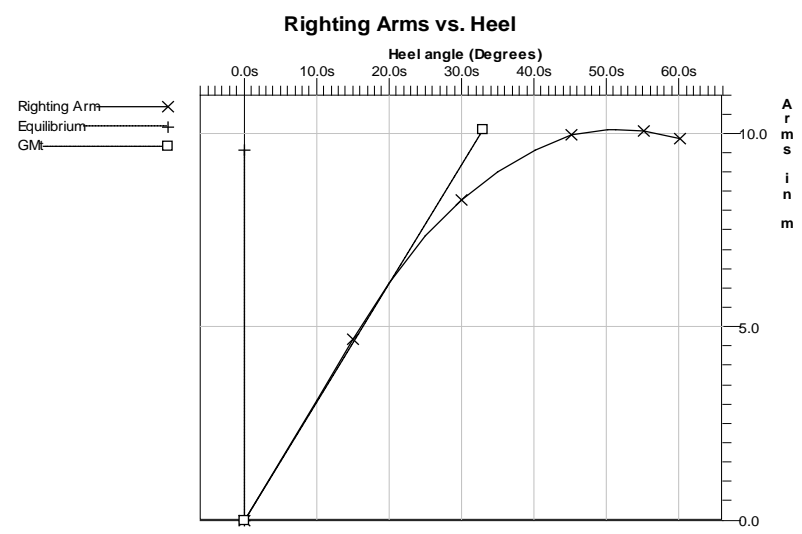

Fig. 13. Ship model righting arm in Autohydro

\section{CONCLUSIONS}

The motion of a ship or floating structure is important for determining of the dynamic load on the crew/ passengers, structural materials and equipment, and cargo. The parameters that influence heaving and pitching amplitude relevant are presented in the study the 97,000 dwt bulk carrier based on Autoship software. Based on regression analysis we estimated amplitude values for various degrees of sea agitation and for various loading situations of the ship. The results are useful for determining additional loads induced both structural elements and the components of equipment and installations on board ships. 
"Mircea cel Batran" Naval Academy Scientific Bulletin, Volume XIX - 2016 - Issue 2 The journal is indexed in: PROQUEST / DOAJ / Crossref / EBSCOhost / INDEX COPERNICUS / DRJI / OAJI I JOURNAL INDEX I I2OR / SCIENCE LIBRARY INDEX / Google Scholar / Academic Keys/ ROAD Open Access I Academic Resources / Scientific Indexing Services / SCIPIO I JIFACTOR

\section{BIBLIOGRAPHY}

[1] Suresh Chandra Misra, Design Principles of Ships and Marine Structures, CRC Press, Dec 1, 2015 Technology \& Engineering - 474 pages online: https://books.google.ro/books?id=80 wCgAAQBAJ\&dq=Moor, + D. + I., + Murdey, + D. + C.,$\&$ source $=$ gbs navlinks $\frac{\mathrm{S}}{[2]}$

[2] M. José Legaz, Computer aided ship design: a brief overview, SeMA Journal December 2015, Volume 72, Issue 1, pp 47-59

[3] Apostolos Papanikolaou, General on Ship Design, Chapter Ship Design pp 1-68

Date: 16 September 2014 Online: http://link.springer.com/chapter/10.1007/978-94-017-8751-2 1

[4] Autoship Software http://cargomanagement.autoship.com/ 